S1 File. Infectious diseases questionnaire. Questionnaire applied during clinical evaluations in original language (Spanish) and English.

\title{
Original language (Spanish)
}

En los últimos 12 meses...

¿... cuántas infecciones respiratorias (anginas, catarros, gripas, gripes, infecciones de garganta, resfriados, sinusitis, etc.) ha padecido?

Número de infecciones |

¿... cuántas infecciones urinarias (ardor al orinar, dolor en la vejiga, urgencia para orinar) ha padecido que hayan requerido antibiótico?

Número de infecciones

¿... cuántas infecciones vaginales (ardor vaginal, comezón, flujo anormal) ha padecido?

Número de infecciones

\section{English}

In the last 12 months...

How many respiratory infections (sore throat, colds, flu, throat infections, colds, sinusitis, etc.) have you had?

Number of infections | _ $\| \ldots$

How many urinary infections (burning when urinating, bladder pain, urge to urinate) that required antibiotics have you had?

Number of infections | _ $\|$

How many vaginal infections (vaginal burning, itching, abnormal discharge) have you had?

Number of infections $\mid \ldots \ldots \ldots$ 